Research Article

\title{
Optimizing Synchronization of Tennis Professional League Live Broadcast Based on Wireless Network Planning
}

\author{
Kai Tang (iD) and Lian-Juan Huo \\ School of Sports Science and Technology, Wuhan Sports University, Wuhan 430205, China \\ Correspondence should be addressed to Kai Tang; tangkai@whsu.edu.cn
}

Received 29 April 2021; Revised 25 May 2021; Accepted 5 June 2021; Published 22 June 2021

Academic Editor: Fazlullah Khan

Copyright (C) 2021 Kai Tang and Lian-Juan Huo. This is an open access article distributed under the Creative Commons Attribution License, which permits unrestricted use, distribution, and reproduction in any medium, provided the original work is properly cited.

\begin{abstract}
The live broadcast of the common league mainly adopts the design of streaming media data block by block. The secondary processing of streaming data is carried out on the server-side. It results in a higher delay during a live broadcast. The higher delay has numerous disadvantages during broadcasting for delay-sensitive applications. We have to solve the delay problems during a live broadcast. Therefore, in this paper, we optimize the synchronization of the tennis professional league live broadcast based on wireless network planning. In the proposed scheme, the 3D detection target model is first constructed, and the background is extracted from the moving video image. The extraction is performed using the interframe difference elimination algorithm, and the target motion trajectory is predicted. Then the phase difference characteristics of the image foreground trajectory are analyzed by the Hilbert transform to detect target missing points. By observing the vertex structure of the target missing point, the phase Fourier transform frame is constructed. Finally, the synchronization of the league live broadcast is encoded and decoded. Based on the timestamp, the synchronization optimization of the tennis professional league live broadcast is completed. The experimental results show that the synchronization optimization method has good synchronization, high resolution, short reaction time, and good detection effect.
\end{abstract}

\section{Introduction}

With the development of science and technology, the carriers and communication platforms for knowledge accumulation have become more and more abundant in modern society. In modern society, as an interactive platform with high efficiency, real-time, and wide dissemination scope, the Internet has gradually become an important force for promoting economic development and social progress after more than 40 years of rapid development [1]. As a medium of rich information, live broadcasting is a special derivative form under the network environment. Because of its excellent network adaptability, it has been given more attention and plays an important role in the network application and social life [2]. Nowadays, live broadcast of the professional league has developed into an important application field of streaming media technology. It has become an indispensable part of sports events because of its rich content and convenient access to information [3].

At present, the main ways to broadcast live events are not limited to live television, live text pictures, live Internet, and studio interviews categories. Among these live broadcasts, professional tennis league live broadcasts have the most intuitive and clear visual effects. The TV live broadcasts are equipped with very professional commentators, which provide an effective way for sports enthusiasts to understand the events and athletes fully. Hence, it is very popular with sports viewers. The reference [4] proposed a mini-league perspective on the impact of premier league broadcasts on audience demand in Denmark. Over the years, extensive research has been conducted on the impact of live football broadcasts on audience demand in Europe. However, very little has been done on small leagues. This paper helps fill this gap by adopting a robust panel data regression model for 2010/11 to 2015/16 at the Danish level. It is discovering that 
games in conflict with premier league broadcasts do not affect spectators. The weather is an essential driver of demand. The reference [5] used the influence of visual training on volleyball players' motor skills. It evaluated the influence of simple cognitive training on sports-related skills. Spike performance is improved after training for such a task when the target is indicated before spike execution but not when the target is indicated during execution. After one month of visual task training, the peak improvement was retained to a certain extent. Based on the above research, the optimization of tennis professional league broadcast synchronization based on wireless network planning is proposed. With the rapid improvement of China's domestic economic level, people's pursuit of material culture and spirit is generally improved. While all kinds of entertainment programs are full of public life and cause aesthetic fatigue, sports events have become another major spiritual pillar that has got more attention. Tennis professional league broadcast brings entertainment and stimulation to the public and can bring some other important role. Due to the important role of competitive sports and the spiritual needs of the public, our country pays more and more attention to live sports events; the level of live broadcasting is higher and higher; and the accumulated live experience is more and more abundant.

The rest of the paper is organized as follows. In Section 2, optimization methods are discussed. In Section 3, stabilization and synchronization mechanisms were elaborated, followed by experimental results and discussion in Section 4 . Finally, Section 5 concludes the paper and provides future research directions.

\section{Optimization Method of Lost Point Feature in Tennis Professional League Live Video}

This section discusses a target missing point detection model, a target missing point detection method, and a feature optimization method of target missing point.

\subsection{Target Missing Point Detection Model. Figure 1 describes} the tennis professional league live video target missing point detection model. The model is established in a three-dimensional coordinate system. The camera is used to collect the tennis player's motion image. The image is parallel to the $x$ - and $y$-axis and perpendicular to the $z$-axis. The $z$-axis plane distance between the highest point of the image and the origin of the three-dimensional coordinate system is $f$. If $(x, y, z)$ is used as the coordinate point of the 3D image, $(x, y)$ represents the coordinate point of the image on the $z$-axis plane, and $\left[\begin{array}{lll}x & y & z\end{array}\right]^{T}$ represents the 3D matrix point of the image.

The model for detecting missing points of the tennis professional league live video object extracts the background from the tennis player's sports image. It predicts the trajectory, then carries on the real-time tracking, and deletes the background to leave behind the foreground containing the target (namely, tennis in motion). It also detects the situation of the missing point of the target. The process based

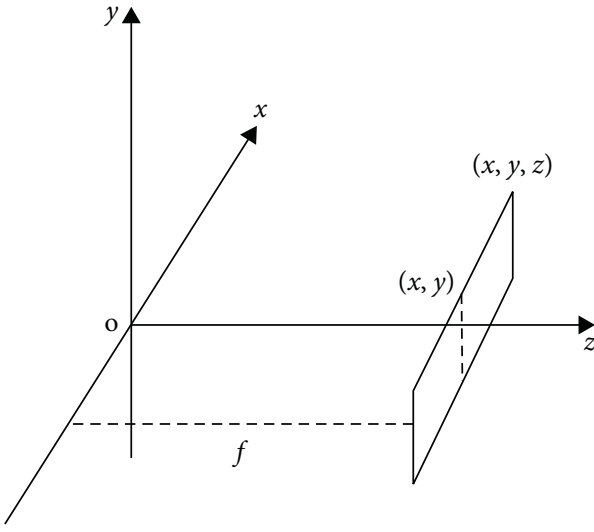

FIGURE 1: Model of detecting missing point in tennis professional league live video.

on wireless network planning is generally divided into three stages: the preliminary preparation for the planned tennis professional league lives broadcast, the pre-planning for the tennis professional league live broadcast, and the detailed planning for the tennis professional league live broadcast $[6,7]$. The tennis professional league broadcast pre-preparation stage needs to complete the analysis of the needs of planning, user business model, and the establishment of radio model correction. The tennis professional league live pre-planning phase of the main coverage needs to complete the wireless network estimates, capacity estimates, and site planning. The detailed planning stage of tennis professional league broadcast mainly completes coverage planning, capacity simulation, and parameter planning. It evaluates the overall performance of wireless network planning through wireless network system simulation [8]. Figure 2 shows the instrumentation model workflow.

As shown in Figure 2, the tennis professional league live video's target missing point detection model carries out different functions, for example, texture extraction, frame value judgment and matching, and motion trajectory prediction successively for the processing of moving image background. Texture extraction is carried out according to different colors and shapes in the image [9]. Frame value judgment and matching need to obtain the similarity between adjacent frames in the image background. According to the similarity level, the image is divided into different frame blocks to form the frame-block matrix $\left[\begin{array}{ccc}x^{*} & y^{*} & z^{*}\end{array}\right]^{T}$. The frame-block matrix is mapped to the plane of $(x, y)$, and the mapping point is $(0,0, f)$. First, the motion displacement and angle of the target in the detection model have assumed to be linear. The relationship between the frame-block matrix $\left[\begin{array}{lll}x^{*} & y^{*} & z^{*}\end{array}\right]^{T}$ and the three-dimensional moving image matrix $\left[\begin{array}{lll}x & y & z\end{array}\right]^{T}$ is as follows:

$$
\left[\begin{array}{l}
X^{*} \\
T^{*} \\
Z^{*}
\end{array}\right]=\left[\begin{array}{lll}
a_{11} & a_{12} & a_{13} \\
a_{21} & a_{22} & a_{23} \\
a_{31} & a_{32} & a_{33}
\end{array}\right]\left[\begin{array}{l}
X \\
Y \\
Z
\end{array}\right]+\left[\begin{array}{l}
b_{1} \\
b_{2} \\
b_{3}
\end{array}\right] .
$$

Let the coordinate point of the frame-block mapping matrix in the $z$-axis plane be $\left(x^{*}, y^{*}\right)$. The $x^{*}$ and $y^{*}$ values 


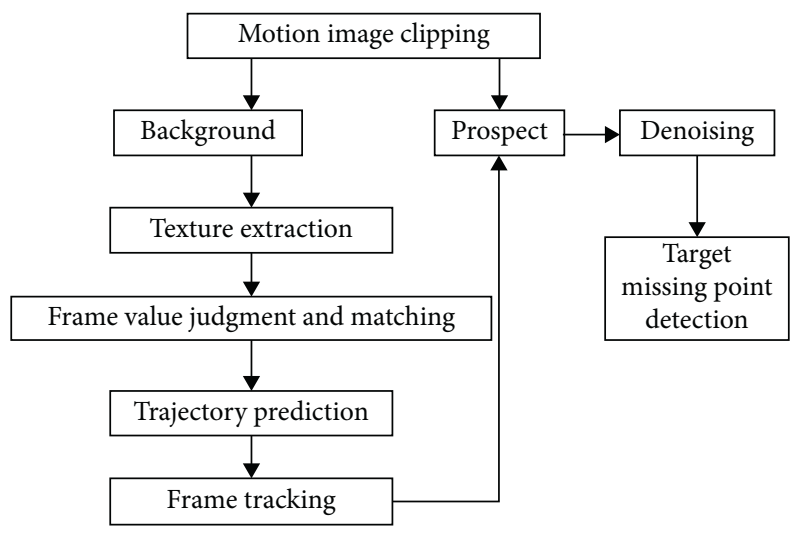

FIGURE 2: Detection model workflow.

can be obtained by the orthogonal projection of Equation (1). The function expression is as follows:

$$
\left\{\begin{array}{l}
x^{*}=a_{1} x+a_{2} y+a_{3} \\
y^{*}=a_{4} x+a_{5} y+a_{6}
\end{array}\right.
$$

where $a_{1}, a_{2}, a_{3}, a_{4}, a_{5}$, and $a_{6}$ are trajectory prediction parameters.

The frame-block mapping matrix is the optimal solution for trajectory prediction of target missing points in live video of professional tennis league. Therefore, it is very important to solve the trajectory prediction parameters accurately. The proposed detection method of target missing points in live video of professional tennis league uses frame-block matching algorithm to solve the trajectory prediction parameters [10]. Let the frame-block matrix sequence be $k$, and the $k$ frame block is matched within the range of the moving image matrix. The vectors of the matching results on the $x$ and $y$-axis of the detection model are $U_{k}$ and $V_{k}$, respectively, and the plane coordinates of the center point of the frameblock are represented by $\left(i_{k}, j_{k}\right)$ as follows:

$$
\begin{aligned}
U_{k} & =a_{1} i_{k}+a_{2} j_{k}+a_{3}, \\
V_{k} & =a_{4} i_{k}+a_{5} j_{k}+a_{6} .
\end{aligned}
$$

By calculating $U_{k}+V_{k} / \partial\left(a_{1}, a_{2}, a_{3}, a_{4}, a_{5}, a_{6}\right)=0$, the trajectory prediction parameters are obtained.

2.2. Target Missing Point Detection Method. After the foreground of the moving image is obtained from the detection model and denoised, the proposed target missing point detection method is used for live videos of professional tennis leagues. It performs the Hilbert transform on the foreground frame to obtain the target missing point signal [11]. It realizes the target missing point detection of live video of professional tennis league by limiting the phase difference. In this paper, the target's missing point signal comprises the tennis trajectory of $\left(x^{*}, y^{*}\right)$ points transformed by the Hilbert transform. The result is expressed by $R(t)$ as follows.

$$
R(t)=\frac{\int_{-\infty}^{+\infty} \mathrm{d} r(w) / t-w \mathrm{~d} w}{\pi},
$$

where $t$ is the target's missing point signal set, $r(w)$ is the conjugate logarithm of $R(t)$, and $w$ is the logarithm of $t$. By analyzing the signal $R(t)$ of the target loss point, we can get the following results:

$$
z(t)=r(t)+\operatorname{jr}(w)=A(t) e^{j \delta(t)},
$$

where $A(t)$ and $\delta(t)$ represent the real-time amplitude and phase of the target loss point signal, respectively. The function expression is as follows:

$$
\left\{\begin{array}{l}
A(t)=\sqrt{r^{2}(t)+r^{2}(w)}, \\
\delta(t)=\arctan \left[\frac{r(w)}{r(t)}\right] .
\end{array}\right.
$$

Suppose the initial phase of the target missing point signal is $\delta_{0}$. In that case, the signal frequency is set to $q_{0}$, and the acquisition frequency of the detection model is $q_{s}$. There are $M$ sampling points in a tennis cycle; $M=\operatorname{round}\left(q_{s} / q_{0}\right)$. If the phase difference of the sampling point trajectory between $M / 2$ and $M / 2+1$ is not less than $2 \pi q_{s} / q_{0}$, the sampling value will be lost at this point, which needs to be optimized.

2.3. Feature Optimization Method of Target Missing Point. 2.3.1. Introduction of Optimization Method of Loss Point in Tennis Professional League Live Video Broadcast

(1) When the vertex of the target missing point presents $C=\left\{x^{1}, x^{2}, \ldots, x^{n}\right\}$ ( $n$ is the number of frames of the missing point) structure and can only change linearly, the target missing point has local stability [12]. If the detection result in the previous section indicates that the time of target missing point is $e+1$, let the forward invariant matrix in $C$ be $X_{e}$, where the frame points in column $s$ are $x^{s}$ and $x^{s} \in C$; the linear change of $X_{e}$ is as follows:

$$
X_{e+1}=\mathrm{BX}_{e}+d_{1},
$$

where $B$ is the optimization parameter, and $d_{1}$ is the maximum optimization error allowed by the method. The objective missing point optimization is to determine the size of the optimization parameter $B$. The matrix form of $\left[\begin{array}{c}X_{e+1} \\ 1\end{array}\right]=\left[\begin{array}{cc}B & d_{1} \\ 0 & 1\end{array}\right]\left[\begin{array}{c}X_{e} \\ 1\end{array}\right]$ is used to express Equation (7), which is further rewritten into $Y_{e+1}=\mathrm{TY}_{e}$ form to facilitate understanding and calculation, $Y_{e+1}=\left[\begin{array}{c}X_{e+1} \\ 1\end{array}\right] T=\left[\begin{array}{cc}B & d_{1} \\ 0 & 1\end{array}\right], Y_{e}=\left[\begin{array}{c}X_{e} \\ 1\end{array}\right]$. $B$ and $d_{1}$ were predicted by the least square method as follows:

$$
B=\min _{B}\left\|Y_{e+1}-\mathrm{TY}\right\|_{\mathrm{eF}},
$$


where $\|*\|_{F}$ stands for the Frobenius norm, which is derived from the Hilbert transformation. It is characterized by simple calculation and obeying the law of arithmetic calculation. Equation (8) can be written as follows after splitting in each coordinate axis of the detection model:

$$
\left\{\begin{array}{l}
B_{x}=Y_{e+1}^{x} Y_{e}^{T}\left(Y_{e}^{T} Y_{e}\right)^{-1} \\
B_{y}=Y_{e+1}^{y} Y_{e}^{T}\left(Y_{e}^{T} Y_{e}\right)^{-1} \\
B_{z}=Y_{e+1}^{z} Y_{e}^{T}\left(Y_{e}^{T} Y_{e}\right)^{-1}
\end{array}\right.
$$

where $Y_{e+1}^{x}, Y_{e+1}^{y}$, and $Y_{e+1}^{z}$ represent the mapping matrix of $Y_{e+1}$ on each coordinate axis of the detection model. At $e$ time, the optimal value of the target loss point of the live video of professional tennis league matches given by the proposed method is $\left[\begin{array}{l}B_{x} \\ B_{y} \\ B_{z}\end{array}\right]\left[\begin{array}{ll}X_{e} & 1\end{array}\right]^{T}$.

(2) When the vertex of the target missing point does not have local stability, the error of the optimized parameter $B$ obtained by the least square method does not meet the requirement of $d_{1}$. At this time, all linearly related frame sets in the target missing point should be found and denoted by $M$, the nonlinear frame set $L=C-M, L=a x n$. The parameter $a$ is predicted by the least square method, and the prediction method is the same as $B$. The optimal value of the target missing point is obtained. The optimized value of the target missing point is filled into the foreground of the tennis motion image. Then the background of the image is restored; the whole motion image is obtained after filtering.

\section{Stabilization of Synchronicity of Live Video of Professional Tennis League}

In this section, we elaborated on various aspects of the stabilization and synchronization of actions from video.

3.1. Phase Fourier Transform Frame. There are some jitter and noise in the live tennis professional league game video captured by the mobile high-altitude camera. Therefore, using the stable frame to stabilize the professional tennis league live video provides the foundation for follow-up synchronization optimization. Video stabilization includes the following steps:

(1) Feature detection and matching: the fast corner detection algorithm is used to find the key points in the live video frame of a professional tennis league. The fast corner detection algorithm is a time- and memory-efficient method for calculating stable video features [13]. Then, the fast retinal key descriptors around the key points are extracted, and the key points in each frame are matched with the corresponding key points in the next frame.
(2) Homography estimation: after feature matching, homography estimation is performed between the corresponding points of two frames. In homography transformation, eight parameters are coded to estimate the translation, rotation, zoom, tilt and view angle transformation at one point. $x$ is set as a point in frame $\mathrm{A}, x^{\prime}$ as a point in frame $\mathrm{B}$, and $H$ as the estimated homography matrix; the relationship between them is expressed as follows:

$$
x^{\prime}=\mathrm{Hx} .
$$

The matrix $H$ is expressed as follows:

$$
H=\left[\begin{array}{ccc}
s \cdot a \cos \theta & s \cdot b \sin \theta & t_{x} \\
s \cdot a \sin \theta & s \cdot b \cos \theta & t_{y} \\
P_{x} & P_{y} & 1
\end{array}\right],
$$

where $S$ is the zoom factor, $a$ and $b$ are the tilt factors, $\sin \theta$ and $\cos \theta$ are the rotation factors, $t_{x}$ and $t_{y}$ are the translation factors, and $P_{x}$ and $P_{y}$ are the angle conversion factors.

(3) Parameter smoothing: The singleton transformation obtained in the above steps describes the camera motion after the first frame and can obtain a cumulative transformation relative to the first frame. Subsequently, the Savitzky-Golay smoothing filter was applied to smooth the motion parameters [14]. Savitzky-Golay filter is a data set, which is processed in a continuous $2 \mathrm{M} 1$ data point, and its fitting polynomial is as follows:

$$
y(n)=\sum_{k=0}^{D} a_{k} n^{k} .
$$

The minimum mean squared error is $\varepsilon_{D}=\sum_{n=-M}^{M}(y(n)-x[n])^{2}$. The coefficient $a_{k}$ of the polynomial can be obtained by minimizing the mean squared error. Then the value of the fitted curve at the center of the data window is taken as the filtered value. The first central point is $y(0)=a_{0}$. Then, with the width of $2 \mathrm{M}+1$, the window is moved, and the fitting process is repeated to smooth all the data. Finally, the filtered sequence $y[n]$ is obtained.

(4) Frame bending: Finally, the calculated motion parameters are bent into each frame to get a stable professional tennis league lives video.

Once a stable professional tennis tournament live video is obtained, the spherical features can be extracted from these frames. The following two features are selected here:

(1) Yellow plane intensity characteristics: Because the tennis ball's color is yellow green, the yellow object on the yellow plane looks white. To this end, this color cue can be used to segment the sphere in the frame [15]. 
In this step, the three color planes are extracted from the image. A matrix representing the yellow intensity is created according to the following equation. A threshold is set to separate the sphere from the background. Figure 1 shows an example of extracting a yellow plane.

$$
Y=g-\frac{r}{1.45}-\frac{b}{1.45},
$$

where $Y$ represents the yellow plane, and $r, g$, and $b$ represent the red, green, and blue planes. As shown in Figure 3, the yellow tennis ball in the full-color live video frame of the professional tennis league looks white in the yellow plane. It is found that when the threshold is 11, the tennis ball can be separated from the yellow plane, and the tennis ball looks like a bright object.

(2) Phase quaternion Fourier transform (PQFT) characteristics: Each pixel of an image is represented as a quaternion, namely, RG color channel, BY color channel, intensity channel, and motion channel. The position of the salient region is obtained by using the phase spectrum of the Fourier transform. Figure 4 shows a significant diagram of the phase Fourier transform. The most prominent object is tennis. Therefore, after computing the salient image of the frame, the sphere can be easily segmented. Here a threshold of 0.5 is set to remove the background pixels less than 0.5 .

3.2. Codec Processing Flow. In this paper, the FFmpeg libavcodec, libavformat library, is mainly used to decode tennis professional league live video. First, the video source device collects tennis professional league live video information through the camera and sound pickup module, then encodes and compresses it through FFmpeg, and then packets it, that is, divides a sequential and continuous data stream into small segments called "packets." After the video and the audio streams are packed, the head of the packet is added before each packet for distinguishing. PTS and DTS should be stamped in the packet header, where PTS is the display timestamp, and DTS is the decoding timestamp. And the data are transferred to the player via the network for playback. The decoding process is as follows: the media stream readout through TCP protocol in the network is separated and decoded by FFmpeg. Finally, the original video data YUV and audio data PCM are obtained. The video is finally played. The tennis professional league live video data collection and playback process is shown in Figure 5.

In this paper, the $\mathrm{H} 264$ algorithm is used for images, and the AAC algorithm is used for audio. The data stream has to be read through the established TCP connection; the image and the audio data have to be separated from the video stream. It stores separated image and audio data in their respective buffer queues. Each connection starts its independent processing threads, reads the frame data from the queue, and decodes it. Then according to the time node, the image is sent to the graphics card, and the audio is sent to the sound card for display and playback. The DTS and PTS of audio and video frames can be obtained by calling image and audio decoding library functions. The synchronization algorithm of the tennis professional league live video designed in this paper mainly depends on DTS and PTS.

\subsection{Timestamp-Based Live Video Synchronization Algorithm} for Tennis Professional League. On the mobile phone side, the tennis professional league live video is decoded into the buffer zone. The synchronous control is carried out between the buffer zone and the control program of tennis professional league live video playing equipment. This paper uses a sampling rate of $44100 \mathrm{~Hz}$. Because the audio sampling rate is fixed, but the video sampling rate is not fixed, so the audio sampling rate is the benchmark.

Mainly, audio, video, and audio synchronization is the use of fixed audio sampling rate characteristics. First, the basic reference clock of audio synchronization is obtained by using library functions. Call the function to decode the image, and the DTS will be stored in the corresponding variable during decoding. The DTS determines when the image is decoded and decodes the current average video frame rate based on the number of video frames in the audio time interval per second. Suppose there is an error, such as the video is earlier than the audio. In that case, the average frame rate is reduced, and if the video is later than the audio, the average frame rate is increased, thereby controlling the synchronization of live tennis professional league video by time-killing.

When we get the PTS value of the live video frame of professional tennis league, we can know the time to display the frame. The frame rate of the video frame at the current time should set to be fixed, and the calculation of the playback start time of the video frame is shown in following equation:

$$
P(I)=\frac{P_{\mathrm{TS}}}{R_{\mathrm{gh}}},
$$

where $P(I)$ is the start time of playback, $P_{T S}$ is the display timestamp, and $R_{g h}$ is the frame rate.

However, in most cases, the frame rate is not fixed, and dynamic prediction is used to solve the frame rate. The start time of the next frame is predicted by estimating the start timestamps of the current frame and the previous frame. The frame rate is the reciprocal of the time difference, and the frame rate of the next frame is dynamically updated to the frame rate. The process of calculating the start time of the next frame is shown in the following equation:

$$
\begin{aligned}
& T_{a}=\frac{P_{a}}{R_{a}}, \\
& T_{b}=\frac{P_{b}}{R_{b}}, \\
& T_{c}=\frac{P_{c}}{R_{c}}=P_{c} \times\left(T_{b}-T_{a}\right), \\
& T_{d}=\frac{P_{d}}{R_{d}}=P_{d} \times\left(T_{c}-T_{b}\right),
\end{aligned}
$$




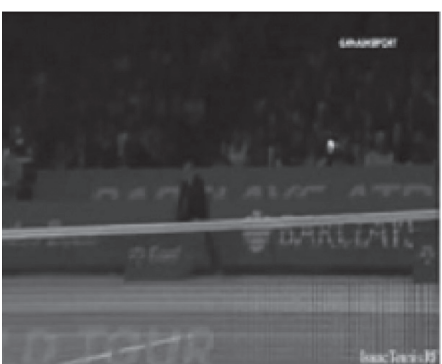

(a)

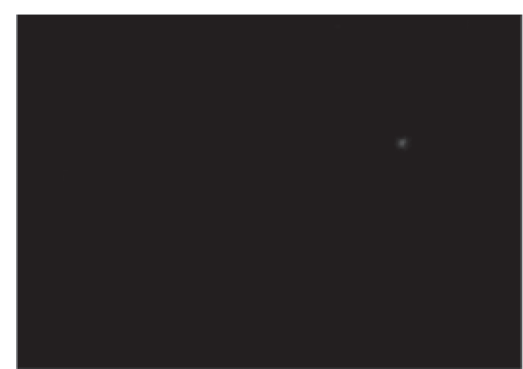

(b)

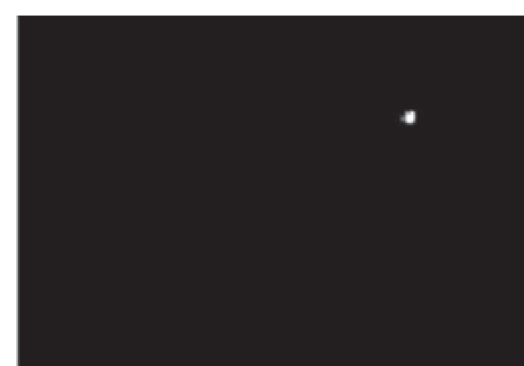

(c)

Figure 3: Different frames: (a) sample frames, (b) frames in the yellow plane, and (c) frames after the threshold are applied.

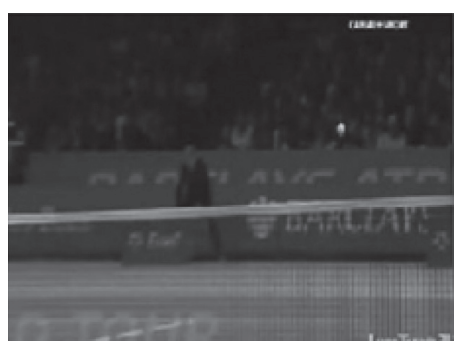

(a)

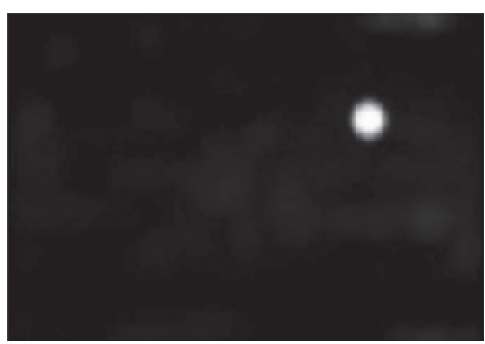

(b)

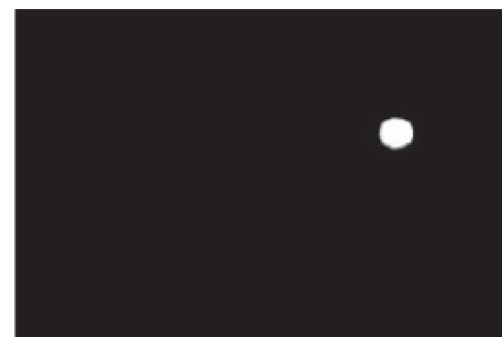

(c)

Figure 4: Frame-salient phase Fourier transform: (a) sample frame, (b) PQFT salient image of the frame, and (c) frame after the threshold is applied.

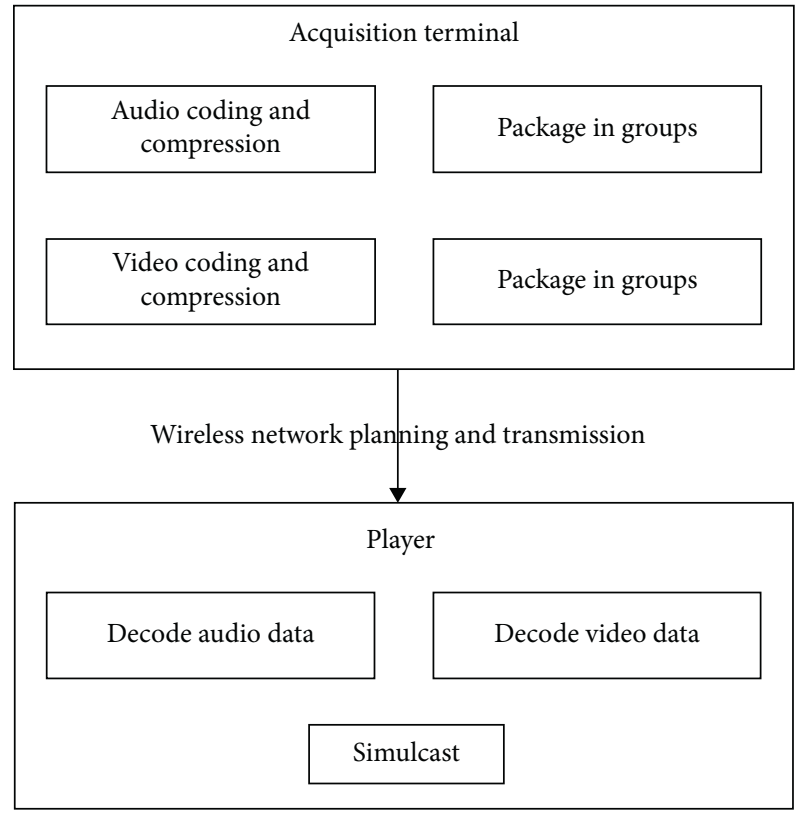

Figure 5: Tennis professional league live video data collection and playback process.

where $P_{a}, P_{b}, P_{c}, P_{d}$ represents the corresponding value of the timestamp, $R_{a}, R_{b}, R_{c}, R_{d}$ represents the frame rate, and different rows represent frames with different serial numbers. First, the display time of the current frame is different from that of the previous frame. The display timestamp of the next frame is equal to multiplying the difference with the next frame.
In order to synchronize the real-time video and audio of the professional tennis league, first, set a refresh function to refresh the video frame and set a key point to refresh the next frame, that is, set the refresh interval Rgh. After the current frame is refreshed, it is necessary to continue to calculate the new refresh interval. The function get_clock () is used to get the playback time, that is, the time of reference clock. The function get_audio_time () is used to get the playback time of the current audio, that is, divide the sample number of the packet obtained by decoding the sampling rate to get the playback time current audio and add it to the reference clock to get the playback time of the current audio. At the same time, the PTS calculation of the live video frame of the professional tennis league is used. We can get the real-time (nonpredicted) start time of the current frame. Then get the delay arrival time of the current frame by subtracting the time twice. If the delay time is positive, it means that the current frame is early, that is, it refreshes the current frame in advance. If it is negative, it means that the current frame is early, and the next refresh delay value will increase. If it is late, the next refresh delay value will decrease. The following formula can be used to update the refresh delay of the refresh function:

$$
R_{\text {time }}=\left(T-C_{\text {time }}\right)+\left(F_{\text {time }}-C_{\text {time }}\right),
$$

where $T$ is the performance time of the next frame, which is predicted by using the display time of the current frame and the display time of the previous frame. It indicates how long it is before the current reference clock, and it needs to be refreshed. It indicates how long the current frame should be delayed to refresh. The $R_{\text {time }}$ is 
TABLE 1: CCTV Grand Slam event commentators in recent four years.

\begin{tabular}{llccc}
\hline Time/year & The Australian Open & The French Open & Wimbledon Championships & The US Open \\
\hline 2012 & Tong Kexin Xu Yang & Zhang Sheng Xu Yang & Tong Kexin Xu Yang & Tong Kexin \\
2013 & Tong Kexin Xu Yang & Zhang Sheng & Tong Kexin Xu Yang & Zhang Sheng Xu Yang \\
2014 & Tong Kexin Xu Yang & Tong Kexin & Zhang Sheng & Tong Kexin \\
2015 & Xu Yang Zhang Sheng & Tong Kexin Xu Yang & Zhang Sheng & Zhang Sheng \\
\hline
\end{tabular}

how long the next frame should be delayed to refresh. After the previous frame is displayed, it needs to recalculate and update the refresh time. Because the network transmission rate limits the mobile live broadcast system, the code and the frame rates are limited in this paper. The refresh time should be set between $66 \mathrm{~ms}$ and $110 \mathrm{~ms}$. Between 9 and 15 frames per second, it is set to $100 \mathrm{~ms}$, which is equal to 0.1 seconds, that is, close to 10 frames per second, to complete the synchronization optimization of tennis professional league live broadcast based on wireless network planning.

\section{Experimental Analysis}

Tennis is a worldwide sports event. Most of the tennis matches broadcast live in the professional league are foreign events. In order to verify the effect and feasibility of optimization of synchronicity of tennis professional league broadcast based on wireless network planning, experiments were conducted. In order to make the experimental data more reasonable, the subjects chosen are all inexperienced or inexperienced novice players. The completely inexperienced tennis players are divided into the second group, and the others are divided into the first group.

The main objective of this experiment is to analyze the effectiveness of the proposed optimization method based on wireless network planning, including the resolution ability, reaction time, and optimization measures. Because the definition of the effectiveness of optimization measures is vague, the experiment describes the effectiveness of optimization measures by analyzing the phase difference of track before and after optimization. The resolution of the target missing points is replaced by the resolution accuracy. The resolution and the reaction time also indirectly express the detection effect of the method.

The experiment was carried out three times. This method's experimental methods, reference [4] method and reference [5] method, and the experiment results were summarized and analyzed. E-PRIME 2.0 is the software used to collect the data of tennis players' sports behavior. It is a kind of software that relies on the psychological principle to convert the information of sports behavior. It has high precision and can construct complicated experimental programs in a short time. It can collect $1 \mathrm{D}$ text data and $2 \mathrm{D}$ and $3 \mathrm{D}$ multimedia data simultaneously [10]. The software used to collect and analyze the collected data is SPSS 22.0.
The commentator understands the English data shown in the referee's voice and on TV during the match in the live broadcast. In the closing stage, the commentator translates the interview with the athlete during the prize presentation. Therefore, as an excellent tennis TV commentator in our country, we must have some foreign language translation ability. There are two modes of personnel composition of tennis TV commentary: two-person and one-person commentary modes. The Grand Slam final events broadcast live on CCTV channel CCTV-5 from 2012 to 2015 is as shown in Table 1.

As can be seen from Table 1, there were nine two-player commentaries and seven single-player commentaries for the 2012-2015 Grand Slam season. Two-person commentators are mainly in the form of commentators' guests. Zhang Sheng, Xu Chang, Tong Kexin and Zhang Sheng are commentators. Most of the commentators are tennis enthusiasts or retired athletes who are familiar with tennis rules and have many years of experience in TV broadcasting or charge of communication. Single commentary mode is only the form of the commentator. In the commentary, to play all the roles and tasks, live synchronization is better.

Based on the statistics and analysis, SPSS 22.0 software results from tennis players' behavior data analysis are described in Table 1. Table 2 summarizes the tennis players' resolution and reaction time to target missing points under the feature optimization of this method, reference [4] method, and reference [5] method.

The data in Table 1 can be analyzed to know the detection effect of three methods on the target loss point of tennis players. Some experiences are better than that of the tennis players with no experience, but there is no big difference. It shows that all three methods can improve the comprehensive quality of tennis players, among which the resolution accuracy of the method in this paper is the highest; the reaction time is the shortest; and the detection effect is the best.

Because there is no significant difference between the two groups of tennis players, only the first group of tennis players is tested to analyze the effectiveness of optimization measures. Figure 6(a) shows the phase difference of the trajectory before optimizing the missing target in a tennis professional league live video. Figure 6(b) is the comparison of the phase difference of the optimized trajectory.

We know that the phase difference of the track reflected by tennis players has been stabilized gradually by analyzing the results. The area of the drop point is smaller that proves that the proposed method shows the best optimization effect and the most effective. 
Table 2: Analysis of tennis player behavior data under different methods.

\begin{tabular}{lccc}
\hline Method name & Analytical project & Group one & Group two \\
\hline \multirow{2}{*}{ Method in this paper } & Resolution accuracy (\%) & 91.42 & 88.64 \\
& Reaction time (ms) & 316.03 & 363.25 \\
\multirow{2}{*}{ Reference [4] method } & Resolution accuracy (\%) & 82.51 & 80.19 \\
& Reaction time (ms) & 373.28 & 296.74 \\
\hline \multirow{2}{*}{ Reference [5] method } & Resolution accuracy (\%) & 70.58 & 66.37 \\
& Reaction time (ms) & 413.21 & 446.59 \\
\hline
\end{tabular}

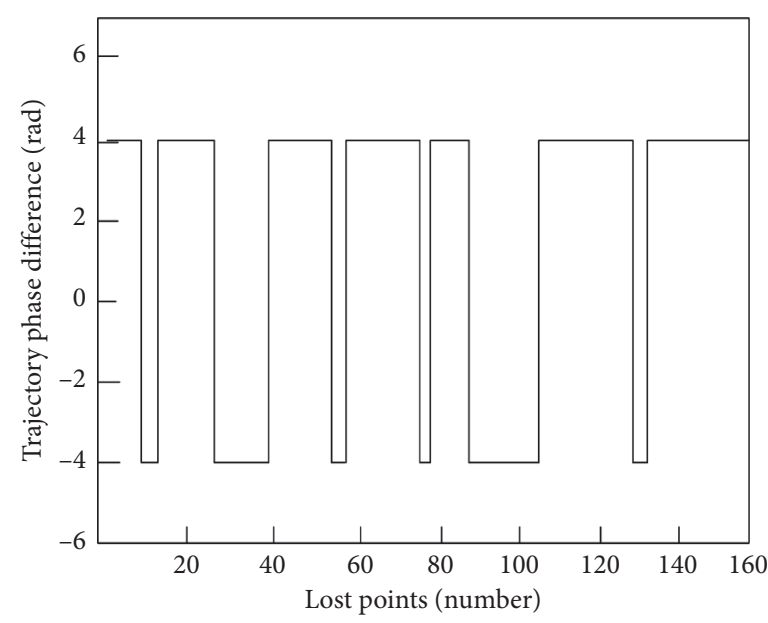

(a)

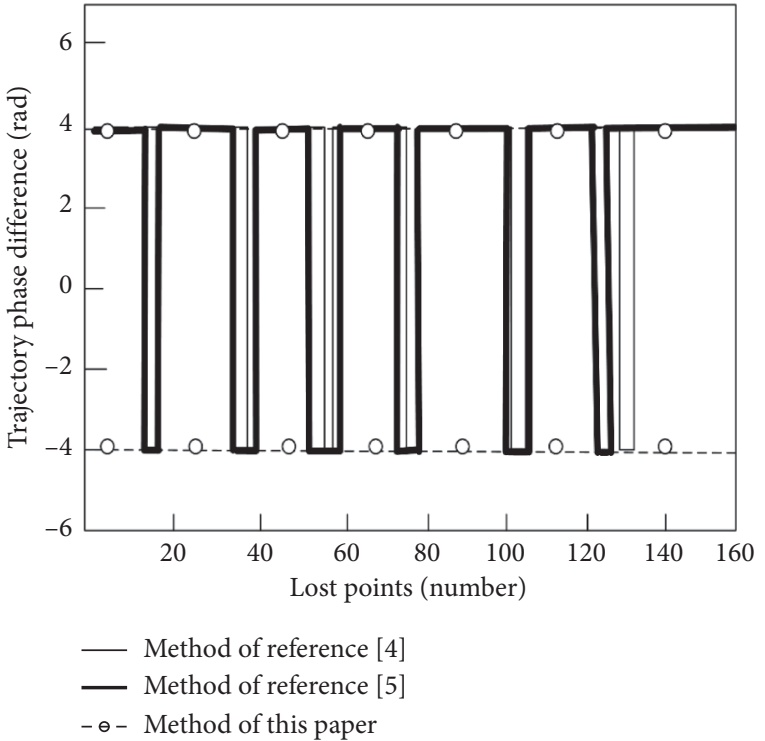

(b)

FIGURE 6: Comparison of the phase difference of trajectory: (a) before optimization and (b) after optimization.

\section{Conclusions and Prospects}

In this section, we discuss the conclusions and the prospects of the study.

\subsection{Conclusions}

(1) In the live broadcast of the tennis league based on wireless network planning, the synchronization results are better than that of existing methods.

(2) The accuracy rate of optimized resolution of live broadcast synchronization of professional tennis leagues is higher. The reaction time is smaller, and the detection effect is fast and efficient.

(3) The optimization effect of live broadcast synchronization of professional tennis leagues based on wireless network planning is the best and most effective than that of the existing methods.

5.2. Prospects. Although some better results have been achieved in this paper, there is room for improvements due to the complexity of the live broadcast system and some other factors.

(1) In the field of mobile live streaming, it is necessary to have a minimum delay in the live streaming systems by dynamically reducing the frame rate and image quality of real-time streaming media in wireless networks.

(2) The functions of the live server of the professional tennis league still need to be enriched. It is possible by optimizing the slicing function of live audio and video data, the live server's distributed deployment, and the load balance among clusters.

(3) In today's increasingly severe network and information security, it is necessary to encrypt professional tennis league lives broadcast data. Simultaneously, multiple security protection and loophole investigations shall be carried out for the system.

\section{Data Availability}

The data sets used and/or analyzed during the current study are available from the corresponding author on reasonable request. 


\section{Disclosure}

The dissertation is a part of the Key Program of 2020 Hubei Educational Science Planning: An Empirical Study on the Teaching Methods and the Quality Enhancement of Tennis Teaching in Universities in Hubei (no. 2020GA079).

\section{Conflicts of Interest}

The authors declare that they have no conflicts of interest.

\section{References}

[1] J. Dvorak and B. M. Pluim, "Injury and illness surveillance in sports: how golf, tennis, cycling and parasport extended the IOC consensus statement to tailor injury and illness surveillance to specific sports," British Journal of Sports Medicine, vol. 55, no. 1, pp. 102-116, 2020.

[2] H. Pas, B. M. Pluim, O. Kilic et al., "Effectiveness of an e-health tennis-specific injury prevention programme: randomised controlled trial in adult recreational tennis players," British Journal of Sports Medicine, vol. 54, no. 17, pp. 101-122, 2020.

[3] G. D. Muynck, B. Soenens, J. Delrue et al., "Strengthening the assessment of self-talk in sports through a multi-method approach," Scandinavian Journal of Medicine \& Science in Sports, vol. 30, no. 3, pp. 25-32, 2020.

[4] C. G. Nielsen, R. K. Storm, and T. G. Jakobsen, "The impact of English premier league broadcasts on Danish spectator demand: a small league perspective," Journal of Business Economics, vol. 89, no. 2, pp. 159-167, 2019.

[5] A. Yz, C. Ctcb, and D. Ngma, "The effects of visual training on sports skill in volleyball players-sciencedirect," Progress in Brain Research, vol. 253, pp. 201-227, 2020.

[6] A. Sluis, M. S. Brink, B. M. Pluim et al., "Self-regulatory skills: are they helpful in the prevention of overuse injuries in talented tennis players?" Scandinavian Journal of Medicine \& Science in Sports, vol. 29, no. 7, pp. 1050-1058, 2019.

[7] R. Butler and P. Massey, "Has competition in the market for subscription sports broadcasting benefited consumers? The case of the English premier league," Journal of Sports Economics, vol. 20, no. 4, pp. 603-624, 2019.

[8] V. D. Alien, "Self-regulatory skills: are they helpful in the prevention of overuse injuries in talented tennis players?" Scandinavian Journal of Medicine \& Science in Sports, vol. 29, no. 7, pp. 1050-1058, 2019.

[9] J. Jarrett, "Gaming the gift: the affective economy of league of legends' fair' free-to-play model," Journal of Consumer Culture, vol. 12, no. 2, pp. 146-159, 2021.

[10] D. Aly, "Broadcast goal senegal football and StarTimes broadcasting deal brings local live football to fans," ChinAfrica, vol. 11, no. 4, pp. 40-41, 2019.

[11] R. Anwit, A. Tomar, and P. K. Jana, "A novel scheme for tour planning of mobile sink in wireless sensor networks," IET Communications, vol. 14, no. 1, pp. 89-95, 2019.

[12] C. Koutroumanides, P. Alexopoulos, A. Laios, and J. Douvis, "The English premier league Internet broadcasting rights selling model-historical study," Gymnasium, vol. 19, no. 2, pp. 44-52, 2019.

[13] Y. Taniguchi, Y. Feng, H. Takamura, and M. Okumura, "Generating live soccer-match commentary from play data," Proceedings of the AAAI Conference on Artificial Intelligence, vol. 33, pp. 7096-7103, 2019.
[14] L. Cervi, "Veni, vidi, Facebooked-live: análisis del éxito de Matteo Salvini en Facebook," Revista CIDOB d'Afers Internacionals, vol. 5, no. 124, pp. 99-122, 2020.

[15] J. A. C. Hattie, "An analysis of in-game feedback provided by coaches in an Australian football league competition," Physical Education and Sport Pedagogy, vol. 25, no. 5, pp. 464-477, 2020. 